\title{
Schur functors and motives
}

\author{
Carlo Mazza* \\ Université Paris 7 - Mathématiques, case 7012 \\ Équipe Topologie et Géométrie Algébriques \\ 2 place Jussieu, 75251 Paris CEDEX 05 \\ mazza@math.jussieu.fr
}

October 20th, 2004

\begin{abstract}
In this article we study the class of Schur-finite motives, that is, motives which are annihilated by a Schur functor. We compare this notion to a similar one due to Kimura. In particular, we show that the motive of any curve is Kimura-finite. This last result has also been obtained by V. Guletskiı̌. We conclude with an example by O'Sullivan of a non Kimura-finite motive which is Schur-finite.
\end{abstract}

If $\lambda$ is a partition of $n$, the Schur functor $S_{\lambda}$ sends a motive $X$ to the direct summand of $X^{\otimes n}$ determined by $\lambda$. We say that $X$ is Schur-finite if it is annihilated by some Schur functor. This definition is due to Deligne (Del02]) who related Schur-finiteness to super-Tannankian categories. In this paper we study Schur-finite objects in several categories, including motives.

Kimura and O'Sullivan have independently defined a stronger notion which we will call Kimura-finiteness. Kimura showed in Kim that if a motive $M$ is Kimura-finite, then any endomorphism of $M$ is either nilpotent or detected by cohomology. Kimura-finiteness has been examined further by Guletskiı and Pedrini (see GP02 and GP03) and by André and Kahn (see AK02]).

In the first part of this paper we define Schur-finiteness and study its properties in the setting of a $\mathbb{Q}$-linear tensor category. In particular, we investigate its behavior with respect to tensor functors and triangles in the derived category of an abelian category with tensor. In the second part we apply this formalism to the category of classical motives and to Voevodsky's category $\mathbf{D M}_{N i s}^{e f f,-}(k, \mathbb{Q})$. We conclude by showing that the motives of all curves are Kimura-finite. This last result has also been obtained by Guletskiı in Gulb. Using a result by O'Sullivan, we produce a motive which is Schur-finite but not Kimura-finite.

The author would like to thank Chuck Weibel for everything he did. Much credit is due to Pierre Deligne, who introduced the definition. We are also grateful to Claudio Pedrini, Luca Barbieri Viale, and Bruno Kahn for precious conversations and their comments. The author would like also to thank the Instituto de Matemáticas of UNAM in Morelia, Mexico for hospitality while this manuscript was prepared.

\footnotetext{
*Partially supported by INdAM "Borse Di Studio Per L'Estero"
} 


\section{Definitions and basic properties}

In this paper, $\mathcal{A}$ will always be a $\mathbb{Q}$-linear tensor category, in the following sense.

Definition 1.1. We say that a symmetric monoidal category $\mathcal{A}$ is a $\mathbb{Q}$-linear tensor category if it satisfies all of the following:

1. $\mathcal{A}$ is additive, pseudo-abelian, and $\mathbb{Q}$-linear,

2. $\otimes$ is $\mathbb{Q}$-bilinear.

Let $F: \mathcal{A} \rightarrow \mathcal{B}$ be a functor between two $\mathbb{Q}$-linear tensor categories. We say that $F$ is a $\mathbb{Q}$-linear tensor functor if it is $\mathbb{Q}$-linear and it respects the symmetric monoidal structures.

Recall that for every partition $\lambda$ of $n$ there is an idempotent $c_{\lambda} \in \mathbb{Q}\left[\Sigma_{n}\right]$ called the Young symmetrizer. If $\Sigma_{n}$ acts on an object $A$ of $\mathcal{A}$, then there is an algebra map $\mathbb{Q}\left[\Sigma_{n}\right] \rightarrow \operatorname{End}(A)$. We will often confuse the element of $\mathbb{Q}\left[\Sigma_{n}\right]$ with the induced endomorphism of a representation. Since $c_{\lambda}^{2}=c_{\lambda}$ and $\mathcal{A}$ is pseudo-abelian, $c_{\lambda}(A)$ is a direct summand of $A$.

Definition 1.2. Let $\mathcal{A}$ be a $\mathbb{Q}$-linear tensor category. The symmetric group $\Sigma_{n}$ acts on $X^{\otimes n}$ for every $X$. For every partition $\lambda$ of $n>0$, we define $S_{\lambda}(X)=c_{\lambda}\left(X^{\otimes n}\right)$. This assignment makes $S_{\lambda}(-)$ into a functor, which we call the Schur functor of $\lambda$. In particular, we define $\operatorname{Sym}^{n}(X)=S_{(n)}(X)$ and $\wedge^{n} X=S_{(1, \ldots, 1)}(X)$.

The following definitions are directly inspired by Del02] and Kim].

Definition 1.3. An object $X$ of $\mathcal{A}$ is called Schur-finite if there is an integer $n$ and a partition $\lambda$ of $n$ such that $X$ is annihilated by the Schur functor of $\lambda$, i.e., $S_{\lambda}(X)=0$. It follows from 1.4 below that $S_{\mu}(X)=0$ for all $\lambda \subseteq \mu$.

An object $X$ of $\mathcal{A}$ is called even (respectively, odd) if there is an $n$ so that $\Lambda^{n} X=0$ (respectively, $S y m^{n} X=0$ ). An object $X$ is called Kimura-finite if there is a decomposition $X=X_{+} \oplus X_{-}$such that $X_{+}$is even and $X_{-}$is odd.

We will say that the category $\mathcal{A}$ is Schur-finite (respectively, Kimura-finite) if all objects of $\mathcal{A}$ are Schur-finite (respectively, Kimura-finite).

Most of the basic results about Schur functors can be proved in the setting of $\mathbb{Q}$-linear tensor categories. Let $H$ be a subgroup of $G$. Then for any irreducible representation $W_{i}$ of $H$ and $V_{j}$ of $G$, we define $\left[\operatorname{res}\left(V_{j}\right): W_{i}\right]$ to be the multiplicity of $W_{j}$ inside the restriction $\operatorname{res}\left(V_{j}\right)$ of $V_{j}$ to $H$. In particular $\operatorname{res}\left(V_{j}\right)=\oplus\left[\operatorname{res}\left(V_{j}\right): W_{i}\right] W_{i}$. By Frobenius reciprocity, the coefficient of $W_{i}$ is the same as $\left[\operatorname{Ind}_{H}^{G}\left(W_{i}\right): V_{j}\right]$.

Let $n_{i}$ be integers so that $n_{1}+\ldots+n_{r}=n$ and consider $\Sigma_{n_{1}} \times \ldots \times \Sigma_{n_{r}} \subseteq$ $\Sigma_{n}$. Let $\mu_{i}$ be a partition of $n_{i}$, and let $V_{\mu_{i}}$ be the corresponding irreducible representation. For every $\lambda$ partition of $n$, and let $V_{\lambda}$ be the corresponding representation. Then we define $\left[\lambda: \mu_{1}, \ldots, \mu_{r}\right]=\left[\operatorname{res}\left(V_{\lambda}\right): V_{\mu_{1}} \otimes \ldots \otimes V_{\mu_{r}}\right]=$ $\left[\operatorname{Ind}\left(V_{\mu_{1}} \otimes \ldots \otimes V_{\mu_{r}}\right): V_{\lambda}\right]$.

Proposition 1.4. (See Del02, 1.6-1.8].) Let $X$ and $Y$ be objects of $\mathcal{A}$, then:

1. $S_{\mu}(X) \otimes S_{\nu}(X) \cong \oplus[\lambda: \mu, \nu] S_{\lambda}(X)$, where the sum is taken over all partitions $\lambda$ of $n=|\mu|+\nu \mid$; 
2. if $S_{\lambda}(X)=0$, then $S_{\mu}(X)=0$ for all $\lambda \subseteq \mu$;

3. $S_{\lambda}(X \oplus Y) \cong \oplus[\lambda: \mu, \nu]\left(S_{\mu}(X) \otimes S_{\nu}(Y)\right)$ where $|\mu|+|\nu|=|\lambda|$;

4. $S_{\lambda}(X \otimes Y) \cong \oplus\left[V_{\mu} \otimes V_{\nu}: V_{\lambda}\right]\left(S_{\mu}(X) \otimes S_{\nu}(Y)\right)$ where $|\mu|=|\nu|=|\lambda|$.

Corollary 1.5. Kimura-finiteness and Schur-finiteness are closed under direct sums and tensor products. Moreover, every Kimura-finite object is Schur-finite.

Proof. Kimura-finiteness and Schur-finiteness are preserved by $\oplus$ and $\otimes$ by 1.4 . For Kimura-finiteness, this was already proven in Kim, 5.11].

Now every Kimura-finite $X$ is a direct sum of two Schur-finite objects. Since Schur-finiteness is closed under direct sums, $X$ is Schur-finite as well.

Recall that if $\mathcal{A}$ is a $\mathbb{Q}$-linear tensor category, then so is the category $\mathcal{A}^{ \pm}$of super-objects of $\mathcal{A}$.

Lemma 1.6. If $\mathcal{A}$ is Schur-finite (respectively, Kimura-finite), then the category $\mathcal{A}^{ \pm}$is also Schur-finite (respectively, Kimura-finite).

Proof. An object of $\mathcal{A}^{ \pm}$is a pair $(V, W)$. Let us write $\overline{\mathbb{1}}$ for $(0, \mathbb{1})$. If $\lambda$ is a partition of $n$, then $S_{\lambda}(V, 0)=\left(S_{\lambda} V, 0\right)$. However $S_{\lambda}(0, V)=\overline{\mathbb{1}}^{n} \otimes\left(S_{\lambda^{\prime}} V, 0\right)$ where $\lambda^{\prime}$ is the transpose of $\lambda$. But Schur-finiteness (respectively, Kimura-finiteness) is closed under direct sums by [1.5] and therefore we have the statement.

Example 1.7. The category $V e c t_{k}^{ \pm}$of finite-dimensional super-vector spaces over a field $k$ of characteristic zero is a $\mathbb{Q}$-linear tensor category. By 1.6 , every object is Kimura-finite. In fact, every vector space is (non-canonically) isomorphic to $\mathbb{1}^{p} \oplus \overline{\mathbb{1}}^{q}$, and $S_{\lambda}\left(\mathbb{\mathbb { 1 }}^{p} \oplus \overline{\mathbb{1}}^{q}\right)=0$ if and only if the partition $\lambda$ contains the rectangle with $p+1$ rows and $q+1$ columns (see [Del02, 1.9]).

Exercise 1.8. Let $X$ be a scheme and let $\mathbf{V} \mathbf{b}_{X}$ be the category of vector bundles over $X$. Each vector bundle is even, so $\left(\mathbf{V b}_{X}\right)^{ \pm}$is Kimura-finite by 1.6

Before we proceed we need a technical lemma.

Lemma 1.9. Let $\mathcal{A}$ be an abelian $\mathbb{Q}$-linear category, and consider a short exact sequence

$$
0 \rightarrow A \rightarrow B \rightarrow C \rightarrow 0
$$

of $\Sigma_{n}$-equivariant maps. Then we have a short exact sequence

$$
0 \rightarrow c_{\lambda}(A) \rightarrow c_{\lambda}(B) \rightarrow c_{\lambda}(C) \rightarrow 0
$$

Proof. Since the Young symmetrizer is an idempotent subfunctor of the identity, a diagram chase yields the result.

Lemma 1.10. (Cf. Del02, 1.19].) Let $\mathcal{A}$ be an abelian $\mathbb{Q}$-linear tensor category where the tensor is right exact. Suppose we have a short exact sequence $0 \rightarrow$ $A \rightarrow B \rightarrow C \rightarrow 0$, and that $B$ is Schur-finite (respectively even, respectively odd). Then $C$ is Schur-finite (respectively even, respectively odd). Moreover, if $A$ and $B$ are flat objects with respect to the tensor product, then $A$ is Schur-finite (respectively even, respectively odd). 
Proof. By 1.9, each $c_{\lambda}\left(B^{\otimes n}\right) \rightarrow c_{\lambda}\left(C^{\otimes n}\right)$ is onto, and, if $A$ and $B$ are flat, each $c_{\lambda}\left(A^{\otimes n}\right) \rightarrow c_{\lambda}\left(B^{\otimes n}\right)$ is into.

The presence of a $\mathbb{Q}$-linear tensor functor between two $\mathbb{Q}$-linear tensor categories creates relations between Schur-finite objects.

Lemma 1.11. Let $F: \mathcal{A} \rightarrow \mathcal{B}$ be a $\mathbb{Q}$-linear tensor functor. If an object $X$ of $\mathcal{A}$ is Schur-finite, so is $F(X)$. If $F$ is also faithful, then the converse holds, i.e., if $F(X)$ is Schur-finite, then so is $X$.

Proof. The result follows from the fact that $F\left(S_{\lambda}(X)\right)=S_{\lambda}(F(X))$ for all objects $X$ of $\mathcal{A}$.

Example 1.12. Lemma 1.11 fails for Kimura-finiteness. Let $\mathcal{A}$ be the category of graded modules over the graded algebra $A=k[\epsilon]$, where $\epsilon^{2}=0$. The forgetful functor $F: \mathcal{A} \rightarrow \operatorname{Vect}_{k}^{ \pm}$sends $A$ to $\mathbb{1} \oplus \overline{\mathbb{1}}$. Hence $A$ is neither even nor odd, but it is also indecomposable and therefore it is not Kimura-finite, even though $F(A)$ is Kimura-finite. However, $A$ is Schur-finite as $S_{(2,2)}(A)=0$ (by 1.7 and 1.11).

Example 1.13. Let $\mathcal{A}$ be the category of finitely generated $R$-modules, where $R$ is a commutative $\mathbb{Q}$-algebra. Then $\mathcal{A}$ is a $\mathbb{Q}$-linear tensor category, and by 1.10 all objects are even. Now consider the category of bounded chain complexes of finitely generated $R$-modules. We have a forgetful functor from $\mathbf{C h}^{b}(\mathcal{A})$ to the category $\mathcal{A}^{ \pm}$of super-objects of $\mathcal{A}$. This forgetful functor is a faithful $\mathbb{Q}$ linear tensor functor. Since $\mathcal{A}^{ \pm}$is Schur-finite by [1.6, we have that $\mathbf{C h}^{b}(\mathcal{A})$ is Schur-finite by 1.11. It need not be Kimura-finite; see 3.2

Remark 1.14. Let $\mathcal{M}_{h}$ be the category of $\mathbb{Q}$-linear motives modulo homological equivalence, for a fixed Weil cohomology $H$. By the Künneth formula, the cohomology yields a faithful $\mathbb{Q}$-linear tensor functor $H: \mathcal{M}_{h} \rightarrow V e c t_{\mathbb{Q}}^{ \pm}$. Since $V e c t_{\mathbb{Q}}^{ \pm}$is Schur-finite by 1.7. $\mathcal{M}_{h}$ is Schur-finite by 1.11. Let $\mathcal{M}_{h}^{ \pm}$be the subcategory of motives of $\mathcal{M}_{h}$ for which the odd and the even part of the decomposition of the diagonal are algebraic. Y. André and B. Kahn in [AK02, 9.2.1c+B.2], and independently P. O'Sullivan, proved that $\mathcal{M}_{h}^{ \pm}$is Kimura-finite.

Let $\mathcal{M}_{n}$ be the category of $\mathbb{Q}$-linear motives modulo numerical equivalence. Since we have a $\mathbb{Q}$-linear tensor functor from $\mathcal{M}_{h}$ to $\mathcal{M}_{n}$ and $\mathcal{M}_{h}$ is Schur-finite, $\mathcal{M}_{n}$ is Schur-finite by 1.11.

Kimura (and O'Sullivan independently) conjectured in [Kim, 7.1] that the category $\mathcal{M}_{r}$ of $\mathbb{Q}$-linear motives modulo rational equivalence is Kimura-finite. This conjecture combined with 1.11 implies that $\mathcal{M}_{h}$ is Kimura-finite.

Theorem 1.15. The category $\mathcal{M}_{n}$ is super-Tannakian, i.e., there exists a field $K$ of characteristic zero and a faithful fibre functor from $\mathcal{M}_{n}$ to Vect ${ }_{K}^{ \pm}$.

Proof. The category $\mathcal{M}_{n}$ is abelian and semi-simple.This in particular implies that the tensor product is exact. This category is also rigid by Jan00, p. 232]. Therefore the result comes from 1.14, Del02, 2.1], and the fact that every commutative ring maps to a field. The faithfulness comes automatically from the rigidity (see [Del02, 0.9]). 


\section{Abelian $\mathbb{Q}$-linear tensor categories}

In this section we assume that the $\mathbb{Q}$-linear tensor category $\mathcal{A}$ is abelian and study how Schur-finiteness behaves with respect to extensions.

The following construction is adapted from [Del02, 1.19] and will be useful to prove that Schur-finiteness is closed under extension of flat objects.

Let $\mathcal{A}$ be an abelian tensor category and let $X$ be the extension

$$
0 \longrightarrow P \longrightarrow X \longrightarrow Q \longrightarrow 0
$$

where $P$ and $Q$ are flat objects. Then we define a $\Sigma_{n}$-equivariant filtration of $X^{\otimes n}$ as follows. The filtration $F_{i}\left(X^{\otimes n}\right)$ will be the subobject generated by all $n$-fold tensor products where $n-i$ factors are copies of $P$ and the remaining $i$ are copies of $X$. To make this precise, we establish some notations.

Definition 2.1. Let $X$ be the extension

$$
0 \longrightarrow P \longrightarrow X \longrightarrow Q \longrightarrow 0
$$

For every pair of numbers $i$ and $j$ so that $i+j=n$, we define

$$
T_{j, i}(P, X)=\operatorname{Ind} d_{\Sigma_{j} \times \Sigma_{i}}^{\Sigma_{n}}\left(P^{\otimes j} \otimes X^{\otimes i}\right) .
$$

The $\Sigma_{j} \times \Sigma_{i}$-equivariant maps $P^{\otimes j} \otimes X^{\otimes i} \rightarrow X^{\otimes n}$ induce $\Sigma_{n}$-equivariant maps $f_{i}: T_{j, i}(P, X) \rightarrow X^{\otimes n}$. We define

$$
F_{i}\left(X^{\otimes n}\right)=\operatorname{Im}\left(f_{i}\right)=T_{j, i}(P, X) /\left(\operatorname{Ker} f_{i}\right) .
$$

In particular $F_{0}\left(X^{\otimes n}\right)=f_{0}\left(T_{n, 0}(P, X)\right)=P^{\otimes n}, F_{n}\left(X^{\otimes n}\right)=f_{n}\left(T_{0, n}(P, X)\right)=$ $X^{\otimes n}$ and

$$
T_{n-1,1}(P, X)=\left(P^{\otimes n-1} \otimes X\right) \oplus\left(P^{\otimes n-2} \otimes X \otimes P\right) \oplus \ldots \oplus\left(X \otimes P^{\otimes n-1}\right) .
$$

Since the maps $f_{i}$ are $\Sigma_{n}$-equivariant, so are the $F_{i}\left(X^{\otimes n}\right)$. Since the map $P^{j+1} \otimes X^{i-1} \rightarrow X^{\otimes n}$ factors through $P^{j} \otimes X^{i}$, then the $\Sigma_{n}$-equivariant map $f_{i-1}$ factors through $f_{i}$, and hence $F_{i-1}\left(X^{\otimes n}\right)=\operatorname{Im}\left(f_{i-1}\right) \subseteq \operatorname{Im}\left(f_{i}\right)=F_{i}\left(X^{\otimes n}\right)$. Therefore the $F_{i}\left(X^{\otimes n}\right)$ form a $\Sigma_{n}$-equivariant filtration of $X^{\otimes n}$.

Proposition 2.2. Let $X$ be an extension of two flat objects $P$ and $Q$ and let $F_{i}\left(X^{\otimes n}\right)=\operatorname{Im}\left(f_{i}\right)$ as in 2.1. Then $F_{i} / F_{i-1} \cong T_{n-i, i}(P, Q)$.

Proof. We are going to proceed by induction on $n=i+j$. For $n=1$ it is clear. Let us suppose that the statement is true for $n-1$ and consider the filtration $F_{*}\left(X^{n-1}\right)$ on $X^{n-1}$ and the given filtration $F_{*}(X)$ on $X$. The tensor product of the two filtrations yields the filtration $F_{*}\left(X^{\otimes n}\right)$ on $X^{\otimes n}$. Set $g r_{i}\left(X^{\otimes n}\right)=F_{i}\left(X^{\otimes n}\right) / F_{i-1}\left(X^{\otimes n}\right)$. Since $X$ is flat, $g r_{*}\left(X^{i-1}\right) \otimes g r_{*}(X)=$ $g r_{*}\left(F_{*}\left(X^{n-1}\right)\right) \otimes g r_{*}\left(F_{*}(X)\right) \cong g r_{*}\left(F_{*}\left(X^{\otimes n}\right)\right)=g r_{*}\left(X^{\otimes n}\right)$ by Bou89, Ex. III.2.6]. But $g r_{i}(X)$ is $P$ when $i=0, Q$ when $i=1$, and 0 otherwise. Therefore

$$
\begin{gathered}
g r_{i}\left(X^{\otimes n}\right) \cong\left(g r_{i}\left(X^{n-1}\right) \otimes P\right) \oplus\left(g r_{i-1}\left(X^{n-1}\right) \otimes Q\right) \\
=\left(T_{n-1-i, i}(P, Q) \otimes P\right) \oplus\left(T_{n-i, i-1}(P, Q) \otimes Q\right)=T_{i, j}(P, Q) .
\end{gathered}
$$

And now to the theorem we advertised before. 
Theorem 2.3. (Cf. Del02, 1.19].) Let $\mathcal{A}$ be an abelian $\mathbb{Q}$-linear tensor category. Then any extension of Schur-finite flat objects is Schur-finite.

Proof. Consider the extension

$$
0 \rightarrow P \rightarrow X \rightarrow Q \rightarrow 0
$$

and the corresponding filtration constructed in 2.1. Choose an integer $n$ and a partition $\lambda$ of $n$ so that $S_{\lambda}(P)=S_{\lambda}(Q)=S_{\lambda}(P \oplus Q)=0$. By 2.2 $\oplus_{i} F_{i} / F_{i-1}=$ $(P \oplus Q)^{\otimes n}$. Since $F_{i} / F_{i-1}$ is $\Sigma_{n}$-invariant, $\oplus c_{\lambda}\left(F_{i} / F_{i-1}\right)=c_{\lambda}\left(\oplus F_{i} / F_{i-1}\right)=$ $c_{\lambda}\left((P \oplus Q)^{\otimes n}\right)=S_{\lambda}(P \oplus Q)=0$ and therefore $c_{\lambda}\left(F_{i} / F_{i-1}\right)=0$ for every $i$. Consider the short exact sequences

$$
0 \rightarrow F_{i-1} \rightarrow F_{i} \rightarrow F_{i} / F_{i-1} \rightarrow 0 .
$$

By [1.9, we have short exact sequences

$$
0 \rightarrow c_{\lambda}\left(F_{i-1}\right) \rightarrow c_{\lambda}\left(F_{i}\right) \rightarrow c_{\lambda}\left(F_{i} / F_{i-1}\right) \rightarrow 0 .
$$

But $c_{\lambda}\left(F_{0}\right)=c_{\lambda}\left(P^{\otimes n}\right)=S_{\lambda}(P)=0$ by hypothesis. Hence we proceed by induction to prove that $c_{\lambda}\left(F_{i}\right)=0$ for every $i$. In particular, $S_{\lambda}(X)=c_{\lambda}\left(F_{n}\right)=$ 0 .

Corollary 2.4. Let $\mathcal{A}$ be an abelian $\mathbb{Q}$-linear tensor category. Let $0 \rightarrow P \rightarrow$ $X \rightarrow Q \rightarrow 0$ be a short exact sequence of flat objects. If $S_{\lambda}(P \oplus Q)=0$, then $S_{\lambda}(X)=0$. In particular, an extension of odd (respectively, even) flat objects is odd (respectively, even).

Proof. This is clear from the proof of 2.3 ,

\section{Chain complexes and derived categories}

In this section we will always assume that $\mathcal{A}$ is an abelian $\mathbb{Q}$-linear tensor category. Consider the category $\mathbf{C}=\mathbf{C h}^{-}(\mathcal{A})$ of bounded below chain complexes. If $M$ and $N$ are two objects, then we define $M \otimes_{\mathbf{C}} N=T o t^{\oplus}(M \otimes N)$. Using this tensor product, $\mathbf{C}$ is an abelian $\mathbb{Q}$-linear tensor category.

It is easy to see that all Schur-finite complexes in $\mathbf{C h}^{-}(\mathcal{A})$ are bounded. Therefore we will be interested in bounded complexes.

Lemma 3.1. If $\mathcal{A}$ is abelian and Schur-finite, then $\mathbf{C h}^{b}(\mathcal{A})$ is Schur-finite.

Proof. Consider the faithful forgetful $\mathbb{Q}$-linear tensor functor $\mathbf{C h}^{b}(\mathcal{A}) \rightarrow \mathcal{A}^{ \pm}$. Since $\mathcal{A}$ is Schur-finite, then so is $\mathcal{A}^{ \pm}$by 1.6 . Then 1.11 yields the result.

The category of chain complexes provides us with another example of an object which is Schur-finite but not Kimura-finite, beside 1.12,

Example 3.2. (B. Kahn) Consider the category of bounded chain complexes of $R$-modules, where $R=\mathbb{Q}[x]$. This is clearly a $\mathbb{Q}$-linear tensor category. Let $M$ be the complex $R \stackrel{x}{\longrightarrow} R$. This complex is irreducible, and is not Kimura-finite because $S y m^{n} M \cong M$ and $\wedge^{n} M \cong M[n-1]$. By 3.1, $M$ is Schur-finite. 
Example 3.3. (P. O'Sullivan) Consider the category of chain complexes of coherent modules for $\mathbb{P}^{1}$. By 1.8 and 3.2 , the complex $\mathcal{O}(1) \stackrel{x}{\longrightarrow} \mathcal{O}$ is Schurfinite but not Kimura-finite. This category has the feature that $\operatorname{End}(\mathcal{O})=k$.

Let $\mathbf{D}=\mathbf{D}^{-}(\mathcal{A})$ be the bounded below derived category and consider the localization functor $q: \mathbf{C} \rightarrow \mathbf{D}$. For simplicity, let us assume that $\mathcal{A}$ has enough projectives to avoid some technical difficulties. In the derived category we define the tensor product of two objects $M$ and $N$ as

$$
M \otimes_{\mathbf{D}} N=\operatorname{Tot}^{\oplus}(P \otimes Q),
$$

where $P$ and $Q$ are two projective resolutions of $M$ and $N$, respectively. (See MVW, Lec. 8].)

With these conventions, if $\mathcal{A}$ is an abelian $\mathbb{Q}$-linear tensor category, then the bounded below derived category $\mathbf{D}$ is a $\mathbb{Q}$-linear tensor category.

Lemma 3.4. If $P$ is a complex of projectives, then $q\left(S_{\lambda}(P)\right)=S_{\lambda}(q(P))$. In particular, if $S_{\lambda}(P)=0$ in $\mathbf{C}$, then $S_{\lambda}(P)=0$ in $\mathbf{D}$. Conversely, if $S_{\lambda}(P)=0$ in $\mathbf{D}$, then $S_{\lambda}(P)$ is acyclic in $\mathbf{C}$.

Proof. Clear from the fact that if $P$ is a complex of projectives, then $q\left(P \otimes_{\mathbf{C}} P\right)=$ $P \otimes_{\mathbf{D}} P$.

Lemma 3.5. Let $\mathcal{A}$ be Schur-finite and let $X$ be a bounded complex with a finite projective resolution. Then $X$ is Schur-finite in $\mathbf{D}^{b}(\mathcal{A})$.

Proof. Let $P$ be a finite projective resolution. By 3.1, there is a $\lambda$ so that $S_{\lambda}(P)=0$ in $\mathbf{C}$. By 3.4, $S_{\lambda}(P)$ is zero in $\mathbf{D}$ as well.

Lemma 3.6. Let $\mathbf{D}$ be the derived category of an abelian $\mathbb{Q}$-linear tensor category $\mathcal{A}$. Then Schur-finiteness has the two out of three property.

Proof. Let us consider the triangle $A \rightarrow B \rightarrow C \rightarrow A[1]$. Without loss of generality, we may assume that $A$ and $B$ are Schur-finite, and we need to prove that $C$ is such. We may replace $A$ by a projective resolution $P$, and similarly we replace $B$ with $Q$. If $f: P \rightarrow Q$, we may assume that $C$ is just cone $(f)$ and we have a short exact sequence

$$
0 \rightarrow Q \rightarrow \text { cone }(f) \rightarrow P[1] \rightarrow 0
$$

We will show that cone $(f)$ is Schur-finite in $\mathbf{D}$, i.e., $S_{\lambda}(\operatorname{cone}(f))$ is acyclic for some $\lambda$.

Choose a partition $\lambda$ of $n$ such that the complexes $S_{\lambda}(P[1]), S_{\lambda}(Q)$, and $S_{\lambda}(Q \oplus P[1])$ are all acyclic. Since $\mathcal{A}$ is an abelian $\mathbb{Q}$-linear tensor category, so is $\mathbf{C}$. Therefore we may use 2.1 to define a $\Sigma_{n}$-equivariant filtration $F_{i}$ of $(\text { cone }(f))^{\otimes n}$ coming from the short exact sequence (11). By [1.9] we have short exact sequences

$$
0 \rightarrow c_{\lambda}\left(F_{i-1}\right) \rightarrow c_{\lambda}\left(F_{i}\right) \rightarrow c_{\lambda}\left(F_{i} / F_{i-1}\right) \rightarrow 0 .
$$

Since the sequence (11) splits in every degree, it still splits degreewise when we tensor with any other object. Therefore the proof of 2.2 goes through to give that $\oplus F_{i} / F_{i-1}=(Q \oplus P[1])^{\otimes n}$. Since each $F_{i} / F_{i-1}$ is $\Sigma_{n}$-invariant, we have that 
$\oplus c_{\lambda}\left(F_{i} / F_{i-1}\right)=c_{\lambda}\left(\oplus\left(F_{i} / F_{i-1}\right)\right)=c_{\lambda}\left((Q \oplus P[1])^{\otimes n}\right)=S_{\lambda}(Q \oplus P[1])$, which is acyclic. This forces all $c_{\lambda}\left(F_{i} / F_{i-1}\right)$ to be acyclic. By hypothesis $c_{\lambda}\left(F_{0}\right)=S_{\lambda}(P)$ is acyclic, and therefore it follows by recursion that each $c_{\lambda}\left(F_{i}\right)$ is acyclic. In particular $c_{\lambda}\left(F_{n}\right)=S_{\lambda}($ cone $(f))$ is acyclic, so $S_{\lambda}(\operatorname{cone}(f))=0$ in $\mathbf{D}$.

Corollary 3.7. Let $\mathbf{D}$ be the derived category of an abelian $\mathbb{Q}$-linear tensor category $\mathcal{A}$ and let $A \rightarrow B \rightarrow C \rightarrow A[1]$ be a triangle. If $S_{\lambda}(A \oplus C)=0$, then $S_{\lambda}(B)=0$. In particular, if $A$ and $C$ are odd (respectively, even), then $B$ is odd (respectively, even.)

Proof. Clear from the proof of 3.6 .

Remark 3.8. Lemma 3.6 does not hold for Kimura-finiteness. Let D be the derived category category of finitely generated $\mathbb{Q}[x]$-modules. We have a triangle $\mathbb{Q}[x] \stackrel{x}{\longrightarrow} \mathbb{Q}[x] \longrightarrow \mathbb{Q} \longrightarrow \mathbb{Q}[x][1]$ but $\mathbb{Q}$ is not Kimura-finite by 3.2.

Example 3.9. Consider the category $S h(X)$ of coherent sheaves on a quasiprojective scheme $X$ over a field containing $\mathbb{Q}$. Replacing "projective" by "locally free", the proofs of 3.43 .7 go through.

Example 3.10. Let $\mathcal{A}=S h_{N i s}\left(\mathrm{Cor}_{k}, \mathbb{Q}\right)$ denote the category of Nisnevich sheaves of $\mathbb{Q}$-modules with transfers on $S m / k$. By [MVW] or [Voe00, p. 206], this is an abelian $\mathbb{Q}$-linear tensor category, and so is $\mathbf{D}^{-}(\mathcal{A})$. Replacing "projective" by "representable", we again see that the proofs of 3.43 .7 go through.

\section{Applications to Chow motives}

For any adequate equivalence relation (see Jan00), we can construct a category of $\mathbb{Q}$-linear motives. They are all $\mathbb{Q}$-linear tensor categories and therefore the notions of Schur-finiteness and Kimura-finiteness make sense (see 1.14 above). Kimura-finiteness has been studied in [Kim, AK02, GP02, GP03, and Gula.

Example 4.1. It is well known that $M\left(\mathbb{P}^{1}\right)=\mathbb{1} \oplus \mathbb{L}$. Clearly, the switch acts as the identity on both $\mathbb{1} \otimes \mathbb{1}$ and $\mathbb{L} \otimes \mathbb{L}$, and therefore $\Lambda^{2} \mathbb{1}=\Lambda^{2} \mathbb{L}=0$. Thus,

$$
S_{\lambda}\left(\mathbb{P}^{1}\right)=0 \text { iff } \lambda \supseteq(1,1,1) .
$$

A similar argument shows that the motive of $\mathbb{P}^{n}$ is Kimura-finite.

We cite the following result without proof.

Proposition 4.2. (See [Kim, Corollary 4.4].) The motive of every smooth projective curve is Kimura-finite.

Lemma 4.3. Let $M$ and $N$ be two motives and let $\lambda$ be a partition of $d$. If $\Lambda^{2}(N)=0$, then $S_{\lambda}(M \otimes N)=S_{\lambda}(M) \otimes N^{\otimes d}$.

Proof. By 1.4

$$
S_{\nu}(M \otimes N)=\oplus C_{\lambda, \eta, \nu} S_{\lambda}(M) \otimes S_{\eta}(N),
$$

where $|\lambda|=|\eta|=|\nu|=d$ and $C_{\lambda, \eta, \nu}=\left[V_{\lambda} \otimes V_{\eta}: V_{\nu}\right]$. In this case, $S_{\eta}(N)=0$ for all partitions $\eta$ of $d$ except for $S_{(d)}(N)=N^{\otimes d}$. But $C_{\lambda,(d), \nu}=C_{\lambda, \nu,(d)}$ which is 1 if $\lambda=\nu$ and it is zero otherwise. The result is proved. 
Corollary 4.4. A motive $M$ is Schur-finite if and only if $M \otimes \mathbb{L}$ is Schur-finite.

Corollary 4.5. Let $X_{Y}$ be the blowup of a smooth projective variety $X$ along a pure codimension $r$ smooth subvariety $Y$. If $M\left(X_{Y}\right)$ is Schur-finite, then both $M(X)$ and $M(Y)$ are Schur-finite. Conversely, if $M(X)$ and $M(Y)$ are both Schur-finite, then $M\left(X_{Y}\right)$ is Schur-finite.

Proof. Just recall from Man68 that if $X_{Y}$ is the blowup of a variety $X$ along a pure codimension $r$ subvariety $Y$, we have that:

$$
M\left(X_{Y}\right)=M(X) \oplus\left(\bigoplus_{i=1}^{r-1} M(Y) \otimes \mathbb{L}^{\otimes i}\right) .
$$

Corollary 4.6. Schur-finiteness is a birational invariant for smooth projective surfaces.

Proof. If two surfaces are birationally equivalent, then there is a sequence of blow-ups and blow-downs along points which connects them.

The Kimura-finite analogues of 4.4, 4.5] and 4.6 were established in [Kim.

\section{Applications to the category DM}

Let $\mathbf{D M}=\mathbf{D M}_{N i s}^{e f,-}(k, \mathbb{Q})$ be the tensor triangulated category of $\mathbb{Q}$-linear motives, i.e., the localization by $\mathbb{A}^{1}$-weak equivalences of the derived category of (cochain) complexes of Nisnevich sheaves $\mathbf{D}^{-}=\mathbf{D}^{-}\left(\operatorname{Sh}_{N i s}\left(\mathrm{Cor}_{k}, \mathbb{Q}\right)\right)$. Recall that the tensor structure is given by the localization of $\otimes_{L, N i s}^{t r}$ (see [MVW] 9.5, 14.2 and 14.22]). We write $q$ for the localization functor $\mathbf{D}^{-} \rightarrow \mathbf{D M}$ and $i$ for the adjoint embedding $\mathbf{D M} \rightarrow \mathbf{D}^{-}$. By $3.10 \mathbf{D}^{-}$is a $\mathbb{Q}$-linear tensor category, and therefore $\mathbf{D M}$ is a $\mathbb{Q}$-linear tensor category as well.

Remark 5.1. By [Voe00, p. 197] we have a faithful $\mathbb{Q}$-linear tensor functor from the category of classical Chow motives $\mathcal{M}_{r}$ to DM. Therefore, proving that a motive is Schur-finite in DM is equivalent to proving it in the category $\mathcal{M}_{r}$ of Chow motives.

Lemma 5.2. Let $M$ be a motive over $k$ and let $K$ be a finite extension of $k$. Let $M_{K}$ be the corresponding motive over $K$. If $M_{K}$ is Schur-finite (respectively, Kimura-finite), then $M$ is Schur-finite (respectively, Kimura-finite).

Proof. Since we are working with $\mathbb{Q}$ coefficients, the proof of [MVW, 1.12] goes through in this setting and we have $\mathbb{Q}$-linear adjoint functors

$$
\operatorname{PreSh}\left(\operatorname{Cor}_{k}, \mathbb{Q}\right) \stackrel{\phi}{\longrightarrow} \operatorname{PreSh}\left(\operatorname{Cor}_{K}, \mathbb{Q}\right) \stackrel{\psi}{\longrightarrow} \operatorname{PreSh}\left(\operatorname{Cor}_{k}, \mathbb{Q}\right),
$$

where $M_{K}=\phi(M)$ and $M$ is a direct summand of $\psi\left(M_{K}\right)$. Since $\psi$ is a $\mathbb{Q}$-linear tensor functor, the result for Schur-finiteness follows from Lemma 1.11 In the Kimura-finite case, we conclude by [GP02, 3.11].

Note that $q$ is a $\mathbb{Q}$-linear tensor functor, but it is not faithful. Therefore if $S_{\lambda}(q A)=0$ in $\mathbf{D M}$, then $S_{\lambda}(A)$ need only be $\mathbb{A}^{1}$-weak equivalent to 0 in $\mathbf{D}^{-}$. Note also that $i$ is not a $\mathbb{Q}$-linear tensor functor. 
Proposition 5.3. Schur-finiteness has the two out of three property in DM.

Proof. Consider the triangle $A \rightarrow B \rightarrow C \rightarrow A[1]$ in DM. We may assume that $A$ and $B$ are Schur-finite, and we need to prove that $C$ is such. Choose an integer $n$ and a partition $\lambda$ of $n$ such that $S_{\lambda}(A[1] \oplus B)=0$ in DM. We will show that $S_{\lambda}(C)=0$ in $\mathbf{D M}$.

Applying $i$ to the triangle above yields a triangle in $\mathbf{D}^{-}$, but $S_{\lambda}(i A[1] \oplus i B)$ may only be $\mathbb{A}^{1}$-weakly equivalent to 0 in $\mathbf{D}^{-}$. Let us replace $A$ and $B$ by quasiisomorphic complexes $P$ and $Q$, respectively, which are sums of representables of the form $\mathbb{Q}_{t r}(X)$ in each degree. If $f: P \rightarrow Q$, we may assume that $C$ is just cone $(f)$ and we have a short exact sequence in $\mathbf{C}=\mathbf{C h}^{-}\left(\operatorname{Sh}_{N i s}\left(\operatorname{Cor}_{k}, \mathbb{Q}\right)\right)$

$$
0 \rightarrow Q \rightarrow \operatorname{cone}(f) \rightarrow P[1] \rightarrow 0
$$

By 3.10 , $\mathbf{C}$ is an abelian $\mathbb{Q}$-linear tensor category. Consider the filtration $F_{*}$ of cone $(f)^{\otimes n}$ given by 2.1. By [1.9, we have short exact sequences in $\mathbf{C}$

$$
0 \rightarrow c_{\lambda}\left(F_{i-1}\right) \rightarrow c_{\lambda}\left(F_{i}\right) \rightarrow c_{\lambda}\left(F_{i} / F_{i-1}\right) \rightarrow 0
$$

We know by hypothesis that $c_{\lambda}\left(F_{0}\right)=c_{\lambda}\left((P[1])^{\otimes n}\right)$ is $\mathbb{A}^{1}$-weakly equivalent to zero. By 2.2, all $c_{\lambda}\left(F_{i} / F_{i-1}\right)$ are $\mathbb{A}^{1}$-weak equivalent to zero. Hence we use induction to conclude that $S_{\lambda}(\operatorname{cone}(f))=c_{\lambda}\left(F_{n}\right)$ is $\mathbb{A}^{1}$-weakly equivalent to zero. But then $S_{\lambda}(\operatorname{cone}(f))=0$ in DM.

Corollary 5.4. The subcategory of DM consisting of Schur-finite objects is thick and closed under twists.

Corollary 5.5. Let $A \rightarrow B \rightarrow C \rightarrow A[1]$ be a triangle in DM. If $S_{\lambda}(A \oplus C)=0$, then $S_{\lambda}(B)=0$. In particular, if $A$ and $C$ are even (respectively, odd) then $B$ is even (respectively, odd).

Proof. Clear from the proof of 5.3

With these results available, we can prove that the motive of every curve is Kimura-finite.

From now on we will write $\mathbb{L}$ for $\mathbb{Q}(1)[2]$ to lighten the notations.

Lemma 5.6. Let $P$ be a smooth rational point on a projective curve $X$. Then the following is a split triangle:

$$
M(X-P) \longrightarrow M(X) \longrightarrow \mathbb{L} \stackrel{0}{\longrightarrow} M(X-P)[1] .
$$

Proof. This is obtained from the triangles on p. 196 in Voe00.

Proposition 5.7. The motive of a smooth curve is Kimura-finite.

Proof. Let $X$ be a smooth curve. There exists a smooth projective curve $\bar{X}$ and an open embedding $X \longrightarrow \bar{X}$, such that the complement is a collection of smooth points $P_{0}, \ldots, P_{n}$. By base change 5.2 we may assume that all the points $P_{i}$ are rational and that $X$ contains a smooth rational point $Q$.

First consider $X^{\prime}=\bar{X}-P_{0}$. By 5.6, we have a triangle

$$
\mathbb{L}[-1] \stackrel{0}{\longrightarrow} M\left(X^{\prime}\right) \longrightarrow M(\bar{X}) \longrightarrow \mathbb{L}
$$


from which we can split off the motive of the rational point $Q$ and, writing $\widetilde{M}(X)$ for the reduced motive $M(X) / M(Q)$ of $X$, get

$$
\mathbb{L}[-1] \stackrel{0}{\longrightarrow} \widetilde{M}\left(X^{\prime}\right) \longrightarrow \widetilde{M}(\bar{X}) \longrightarrow \mathbb{L}
$$

Since $\bar{X}$ is a smooth projective curve, the reduced motive decomposes as $\widetilde{M}(\bar{X})=$ $M_{1}(X) \oplus \mathbb{L}$, where $M_{1}(\bar{X})$ is odd. By [5.6] we may split off the copy of $\mathbb{L}$, and get that $M_{1}(\bar{X}) \cong \widetilde{M}\left(X^{\prime}\right)$. Since $M_{1}(\bar{X})$ is odd, so is $\widetilde{M}\left(X^{\prime}\right)$.

By [Voe0, p. 196], we have a triangle

$$
\oplus_{1}^{n} \mathbb{L}[-1] \longrightarrow M(X) \longrightarrow M\left(X^{\prime}\right) \longrightarrow \oplus_{1}^{n} \mathbb{L}
$$

Splitting off the motive of the point $Q$, we get

$$
\oplus_{1}^{n} \mathbb{L}[-1] \longrightarrow \widetilde{M}(X) \longrightarrow \widetilde{M}\left(X^{\prime}\right) \longrightarrow \oplus_{1}^{n} \mathbb{L}
$$

But $\widetilde{M}\left(X^{\prime}\right)$ is odd by the first part of this proof and clearly $\oplus_{1}^{n} \mathbb{L}[-1]$ is odd, so by $5.5 \widetilde{M}(X)$ is odd too. But $M(X)=\mathbb{1} \oplus \widetilde{M}(X)$, and therefore we have the statement.

Theorem 5.8. The motive of any curve is Kimura-finite.

Proof. The smooth case was established in 5.7. Suppose that $X$ is a singular affine curve. Let $Z$ be the singular locus of $X$, and let $X^{\prime}$ be the normalization. Then we have the cartesian diagram

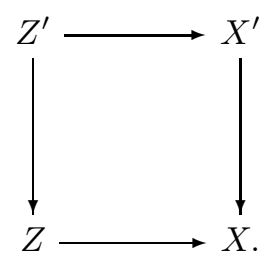

By [Voe0, Prop. 4.1.3], we have a triangle

$$
M\left(Z^{\prime}\right) \rightarrow M(Z) \oplus M\left(X^{\prime}\right) \rightarrow M(X) \rightarrow M\left(Z^{\prime}\right)[1] .
$$

By base change 5.2 we may assume that both $Z$ and $Z^{\prime}$ consist of rational points. Let $K$ be the kernel of the map $M\left(Z^{\prime}\right) \rightarrow M(Z)$ and note that $M\left(Z^{\prime}\right) \cong$ $K \oplus M(Z)$. Then the triangle becomes

$$
K \rightarrow M\left(X^{\prime}\right) \rightarrow M(X) \rightarrow K[1] .
$$

By base change 5.2, we may assume that $X$ contains a smooth rational point which we can split off, and get a triangle

$$
K \rightarrow \widetilde{M}\left(X^{\prime}\right) \rightarrow \widetilde{M}(X) \rightarrow K[1] .
$$

By the proof of [5.7, $\widetilde{M}\left(X^{\prime}\right)$ is odd. But $K[1]$ is also odd, because $M\left(Z^{\prime}\right)$ is even, and therefore $\widetilde{M}(X)$ is odd by 5.5

Now let $X$ be a projective singular curve. By base change 5.2 we may assume that $X$ has a rational point $P$. By [5.6. $M(X)=M(X-P) \oplus \mathbb{L}$. But $X-P$ is an affine curve, and we have seen above that it is Kimura-finite. Therefore $M(X)$ is Kimura-finite. 
Remark 5.9. V. Guletskiü has independently obtained this result (and also 5.5) in his recent preprint Gulb.

Let $\mathbf{D} \mathbf{M}_{g m}=\mathbf{D M}_{g m}^{e f f}(k, \mathbb{Q})$ be the category of effective geometrical motives (see [Voe00, 2.1.1]). Recall that there is a fully faithful $\mathbb{Q}$-linear tensor functor from $\mathbf{D} \mathbf{M}_{g m}$ to $\mathbf{D M}$ and that $\mathbf{D} \mathbf{M}_{g m}$ contains the motives of all smooth schemes. Let $d_{\leq i}=d_{\leq i} \mathbf{D M}_{g m}^{e f f}(k, \mathbb{Q})$ be the thick subcategory of $\mathbf{D M}_{g m}$ generated by the motives of all smooth schemes $X$ of dimension less or equal to $i$ (cf. Voe00, p. 215]).

By 5.3 and 1.4 the category $d_{\leq i}$ is Schur-finite if and only if every smooth motive $M(X)$ is Schur-finite $(\operatorname{dim} X \leq i)$. This observation, together with 5.7, implies the following statement.

Corollary 5.10. The category $d_{\leq 1}$ is Schur-finite.

Remark 5.11. F. Orgogozo proved in Org04 that $d_{\leq 1}$ is equivalent to $\mathbf{D}^{b}\left(1-\operatorname{mot}_{\mathbb{Q}}\right)$, the bounded derived category of 1-motives modulo isogenies. P. O'Sullivan proved that all objects of $\mathbf{D}^{b}\left(1-\right.$ mot $\left._{\mathbb{Q}}\right)$ are Kimura-finite (in $\mathbf{D} \mathbf{M}_{g m}$ ) using the weight filtration on 1-motives. This implies that $d_{\leq 1}$ is actually Kimura-finite.

Recall from [Voe00, 4.3.7] that every object $A$ in $\mathbf{D} \mathbf{M}_{g m}$ has a dual $A^{*}=$ $\underline{\operatorname{Hom}}_{\mathrm{DM}}(A, \mathbb{Z})$, where $\underline{\text { Hom }}_{\mathrm{DM}}$ is the internal Hom-object of DM. By [Voe00], every variety has also a motive with compact support $M^{c}(X)$ associated to it. If $X$ is proper, then $M(X) \cong M^{c}(X)$. If $X$ is smooth of dimension $d$, then $M^{c}(X) \cong M(X)^{*}(d)[2 d]$.

Lemma 5.12. The subcategory of $\mathbf{D M}_{g m}$ consisting of Schur-finite objects is thick and closed under duals and twists.

Proof. The subcategory is thick and closed under twists by [5.4 If $M$ is an object of $\mathbf{D M}_{g m}$ then $\left(S_{\lambda}(M)\right)^{*} \cong S_{\lambda}\left(M^{*}\right)$ by [Del02, 1.18]. In particular $M$ is Schur-finite if and only if $M^{*}$ is so.

Before we state our results, let us investigate further the structure of the categories $d_{\leq i}$. We will write $D_{\leq n}$ for the thick subcategory of $\mathbf{D M}_{g m}$ generated by the motives of all smooth projective varieties of dimension at most $n$. If the ground field admits resolution of singularities, then we have the following facts.

Lemma 5.13. Assume that the ground field $k$ admits resolution of singularities in dimension $\leq n$. If $X$ is a projective variety of dimension less or equal to $n$, then $M(X)^{*}(n)[2 n]=M(X)^{*} \otimes \mathbb{L}^{n}$ is in $D_{\leq n}$.

Proof. We will proceed by induction on $d=\operatorname{dim} X$. If $M$ is in $D_{\leq d}$ then $M \otimes \mathbb{L}^{n-d}$ is in $D_{\leq n}$, so we may assume $d=n$. The case $n=0$ is clear. Let us assume that the statement holds for all varieties of dimension $n-1$ or less. Let $Z$ be the singular locus of $X$. Using resolution of singularities we have a smooth projective variety $X^{\prime}$ and a triangle

$$
M\left(Z^{\prime}\right) \rightarrow M\left(X^{\prime}\right) \oplus M(Z) \rightarrow M(X) \rightarrow M\left(Z^{\prime}\right)[1] .
$$

Dualizing and tensoring with $\mathbb{L}^{n}$ we have the following triangle

$M(X)^{*} \otimes \mathbb{L}^{n} \rightarrow\left(M\left(X^{\prime}\right)^{*} \otimes \mathbb{L}^{n}\right) \oplus\left(M(Z)^{*} \otimes \mathbb{L}^{n}\right) \rightarrow M\left(Z^{\prime}\right)^{*} \otimes \mathbb{L}^{n} \rightarrow M(X)^{*} \otimes \mathbb{L}^{n}[1]$. 
Both $Z$ and $Z^{\prime}$ are of lower dimension, so $M(Z)^{*} \otimes \mathbb{L}^{n}$ and $M\left(Z^{\prime}\right)^{*} \otimes \mathbb{L}^{n}$ are in $D_{<n}$ by induction. But since $X$ is smooth and projective of dimension $n$, $M(X)^{*}(n)[2 n]=M(X)$, which is in $D_{\leq n}$ by definition. By the two out of three property, $M(X)^{*} \otimes \mathbb{L}^{n}$ is in $D_{\leq n}$ as well.

Proposition 5.14. If $k$ admits resolution of singularities in dimension $\leq n$, then the category $d_{\leq n}$

1. contains $M(X)$ and $M^{c}(X)$ for every variety $X$ with $\operatorname{dim} X \leq n$;

2. is equal to $D_{\leq n}$, i.e., it is generated by the motives of smooth projective varieties of dimension $\leq n$.

Proof. We will proceed by induction. The case $n=0$ is clear; let us assume that the statement holds for $n-1$.

Let us prove the first statement. Let $X$ be an $n$-dimensional variety and let $Z$ be a divisor containing its singular locus. Using resolution of singularities, we know that there exist a smooth $X^{\prime}$ and a proper map $p: X^{\prime} \rightarrow X$ which is an isomorphism outside $Z$. From Voe00, Prop. 4.1.3], we have an exact triangle

$$
M\left(Z^{\prime}\right) \rightarrow M(Z) \oplus M\left(X^{\prime}\right) \rightarrow M(X) \rightarrow M\left(Z^{\prime}\right)[1] .
$$

Since both $Z$ and $Z^{\prime}$ are of lower dimension, then $M(Z)$ and $M\left(Z^{\prime}\right)$ are in $d_{\leq n}$ by induction. But $X^{\prime}$ is smooth and therefore $M\left(X^{\prime}\right)$ is in $d_{\leq n}$. By thickness, we conclude that $M(X)$ is in $d_{<n}$ as well.

The proof for the motives with compact support is now elementary. For every $X$, consider a projective closure $\bar{X}$ and the complement $Z=\bar{X}-X$. We have a triangle

$$
M^{c}(Z) \rightarrow M^{c}(\bar{X}) \rightarrow M^{c}(X) \rightarrow M^{c}(Z)[1] .
$$

Since $\bar{X}$ is projective, $M^{c}(\bar{X})=M(\bar{X})$ and $M^{c}(Z)=M(Z)$. But $M(\bar{X})$ is in $d_{\leq n}$ by the first part of this proof, and $M(Z)$ is in $d_{\leq n}$ by induction. By thickness, $M^{c}(X)$ is in $d_{\leq n}$ as well.

And now we prove the second statement. Clearly, $D_{\leq n} \subseteq d_{\leq n}$ and we need to prove that $M(X)$ is in $D_{\leq n}$ for every smooth $X, n=\operatorname{dim} X$. Using resolution of singularities, we may embed $X$ into a smooth projective variety $\bar{X}$. Let $Z$ be the complement $\bar{X}-X$, and consider the triangle

$$
M(X) \rightarrow M(\bar{X}) \rightarrow M^{c}(Z)^{*}(n)[2 n] \rightarrow M(X)[1] .
$$

Since $Z$ is projective, $M^{c}(Z)^{*}(n)[2 n]$ is in $D_{\leq n}$ by 5.13 . Since $M(\bar{X})$ is in $D_{\leq n}$, we conclude by thickness that $M(X)$ is in $D_{\leq n}$.

The following corollaries are obtained from 5.14 and 5.3

Corollary 5.15. If the motive of every smooth projective surface is Schur-finite, then the motive of every surface is Schur-finite.

Example 5.16. The proof shows that if $U$ is an open subset of a projective surface $X$, and $M(X)$ is Schur-finite, then $M(U)$ is Schur-finite.

Corollary 5.17. Assume that $k$ admits resolution of singularities in dimension $\leq n$. If the motive of every smooth projective variety of dimension less or equal to $n$ is Schur-finite, then the motive of every variety of dimension less or equal to $n$ is Schur-finite. 


\subsection{An example of a motive which is not Kimura-finite}

This subsection is based on a private communication from O'Sullivan. We will show that there is a smooth surface $U$ whose motive is Schur-finite but not Kimura-finite.

Theorem 5.18. (O'Sullivan) Let $X_{0}$ be a connected, smooth, and projective surface over an algebraically closed field $k_{0}$ such that $q=0$ and $p_{g}>0$. Let $k=k_{0}\left(X_{0}\right)$ be the function field of $X_{0}$ and let $x_{0}$ be a $k_{0}$-point of $X_{0}$. Let $z$ be the zero-cycle which is the pullback of the cycle $\Delta\left(X_{0}\right)-\left(x_{0} \times X\right)$ along $X_{0} \times k \rightarrow X_{0} \times X_{0}$, and write $Z$ for the support of $z$. Let $U$ be the complement of $Z$ in $X=X_{0} \times k$. Then $M(U)$ is not Kimura-finite.

Let $X$ be any connected, smooth, and projective surface. Let $Z$ be a subset of $n k$-rational points on $X$, and let $U=X-Z$. Then from [Voe00, p. 196], we have a distinguished triangle

$$
M(Z)(2)[3] \rightarrow M(U) \rightarrow M(X) \rightarrow M(Z)(2)[4]
$$

The motive of $X$ decomposes as $M(X)=\mathbb{1} \oplus h_{1}(X) \oplus h_{2}(X) \oplus h_{3}(X) \oplus \mathbb{L}^{\otimes 2}$ and it is known that the composite $\mathbb{L}^{\otimes 2} \rightarrow M(X) \rightarrow M(Z)(2)[4] \cong \oplus_{n} \mathbb{L}^{\otimes 2}$ is the diagonal map, so we may split off one copy of $\mathbb{L}^{\otimes 2}$. This yields the following triangle:

$$
\oplus_{n-1} \mathbb{L}^{\otimes 2}[-1] \rightarrow M(U) \rightarrow \mathbb{1} \oplus h_{1}(X) \oplus h_{2}(X) \oplus h_{3}(X) \rightarrow \oplus_{n-1} \mathbb{L}^{\otimes 2} .
$$

If $q=0$ in $X$, the Picard and the Albanese varieties vanish, and then $h_{1}(X)=$ $h_{3}(X)=0$. So we have:

$$
\oplus_{n-1} \mathbb{L}^{\otimes 2}[-1] \longrightarrow M(U) \longrightarrow \mathbb{1} \oplus h_{2}(X) \stackrel{\partial}{\longrightarrow} \oplus_{n-1} \mathbb{L}^{\otimes 2}
$$

We need the following technical lemma to prove 5.18 .

Lemma 5.19. (O'Sullivan) Let $\mathcal{D}$ be a $\mathbb{Q}$-linear rigid tensor triangulated category with $t$-structure and associated cohomological functor $H_{\tau}^{*}$ with Tannakian heart and let

$$
A \stackrel{f}{\longrightarrow} B \longrightarrow C \longrightarrow A[1]
$$

be a distinguished triangle. Suppose that $A$ and $B$ are both even (or both odd) and that $C$ is Kimura-finite. Then if $H_{\tau}^{*} f=0$ then $f=0$ in $\mathcal{D}$.

Proof of 5.18. Let $\mathcal{D}$ be the derived category of $l$-adic sheaves over Spec $k$ (see [Eke90]). There is a $\mathbb{Q}$-linear tensor functor $R \Gamma$ from $\mathbf{D M}$ to $\mathcal{D}$ associated to $l$-adic cohomology (see Hub00 and Hub04). If we prove that the image of a motive in $\mathcal{D}$ is not Kimura-finite, it will prove that the motive itself is not Kimura-finite in DM.

It is known that $\mathcal{D}$ has a t-structure whose heart is the (Tannakian) category of $l$-adic sheaves. Moreover, $l$-adic cohomology is the composition of $R \Gamma$ with the cohomological functor $H_{\tau}^{*}$ associated to this t-structure. Consider the image of triangle (2) in $\mathcal{D}$. It is known that $\partial$ induces the zero map on $l$-adic cohomology, i.e., $H_{\tau}^{*} R \Gamma(\partial)=0$. Therefore we only need to show that $f=R \Gamma(\partial) \neq 0$ and apply lemma 5.19, to prove that $M(U)$ is not Kimura-finite in DM. 
To prove that $R \Gamma(\partial) \neq 0$ we proceed as follows. Since $p_{g}>0$, the map $H^{2}\left(X, \mathbb{Q}_{l}\right) \rightarrow H^{2}\left(\operatorname{Spec} k, \mathbb{Q}_{l}\right)$ is not zero, and so is the composition

$\varphi: \mathbb{Q}_{l}(-2) \rightarrow H^{2}\left(X, \mathbb{Q}_{l}\right) \otimes H^{2}\left(X, \mathbb{Q}_{l}\right) \rightarrow H^{2}\left(X, \mathbb{Q}_{l}\right) \otimes H^{2}\left(\operatorname{Spec} k, \mathbb{Q}_{l}\right) \rightarrow H^{4}\left(X, \mathbb{Q}_{l}\right)$.

But $\operatorname{Hom}_{\text {Vect } \mathbb{Q}_{l}}\left(\mathbb{Q}_{l}(-2), H^{4}\left(X, \mathbb{Q}_{l}\right)\right) \cong \operatorname{Hom}_{\mathcal{D}}\left(R \Gamma\left(\mathbb{L}^{\otimes 2}\right), R \Gamma(M(X))\right)$ and therefore $\varphi$ defines a non-zero map $\psi: R \Gamma\left(\mathbb{L}^{\otimes 2}\right) \rightarrow R \Gamma(M(X))$. Since $\psi$ factors through $j: R \Gamma\left(\oplus_{n} \mathbb{L}^{\otimes 2}\right) \rightarrow R \Gamma(M(X))$, we have that $j \neq 0$. Thus $R \Gamma(\partial) \neq 0$.

Corollary 5.20. Let $X_{0}$ be a Kummer surface in 5.18. Then, using the notations of [5.18, $M(U)$ is Schur-finite but it is not Kimura-finite. Moreover, the two out of three property does not hold for Kimura-finiteness.

Proof. The scheme $X_{0}$ satisfies the condition of theorem 5.18, therefore $M(U)$ is not Kimura-finite. However, from [GP03, Theorem 11 (ii)], $M\left(X_{0}\right)$ is Kimurafinite, and so is $M(X)$. We conclude by applying 5.3 to (2).

\section{References}

[AK02] Y. André and B. Kahn, Nilpotence, radicaux et structures monoïdales, Rend. Sem. Mat. Univ. Padova 108 (2002), 107-291, with an appendix by P. O'Sullivan. MR 1956434

[Bou89] N. Bourbaki, Algebra. I. Chapters 1-3, Elements of Mathematics, Springer-Verlag, Berlin, 1989, Translated from the French, Reprint of the 1974 edition. MR 90d:00002

[Del02] P. Deligne, Catégories tensorielles, Mosc. Math. J. 2 (2002), no. 2, $227-$ 248 , Dedicated to Yuri I. Manin on the occasion of his 65 th birthday. MR 1944506

[Eke90] T. Ekedahl, On the adic formalism, The Grothendieck Festschrift, Vol. II, Progr. Math., vol. 87, Birkhäuser Boston, Boston, MA, 1990, pp. 197-218. MR 92b:14010

[GP02] V. Guletskiı and C. Pedrini, The Chow motive of the Godeaux surface, Algebraic geometry, de Gruyter, Berlin, 2002, pp. 179-195. MR 1954 064

[GP03] V. Guletski1 and C. Pedrini, Finite-dimensional motives and the conjectures of Beilinson and Murre, K-Theory 30 (2003), no. 3, 243-263, Special issue in honor of Hyman Bass on his seventieth birthday. Part III. MR MR2064241

[Gula] V. Guletskiǔ, A remark on nilpotent correspondences, Preprint, January 27, 2004, K-theory Preprint Archives, http://www.math.uiuc.edu/Ktheory $/ 0651 /$.

[Gulb] —, Finite dimensional objects in distinguished triangles, Preprint, January 5, 2004, K-theory Preprint Archives, http://www.math.uiuc.edu/K-theory/0637/. 
[Hub00] A. Huber, Realization of Voevodsky's motives, J. Algebraic Geom. 9 (2000), no. 4, 755-799. MR 2002d:14029

[Hub04] _ Corrigendum to: "Realization of Voevodsky's motives" [J. Algebraic Geom. 9 (2000), no. 4, 755-799], J. Algebraic Geom. 13 (2004), no. 1, 195-207. MR 2008720

[Jan00] U. Jannsen, Equivalence relations on algebraic cycles, The arithmetic and geometry of algebraic cycles (Banff, AB, 1998), NATO Sci. Ser. C Math. Phys. Sci., vol. 548, Kluwer Acad. Publ., Dordrecht, 2000, pp. 225-260. MR 2001f:14016

[Kim] S.-I. Kimura, Chow motives can be finite-dimensional, in some sense., To appear in J. of Alg. Geom.

[Man68] Ju. Manin, Correspondences, motifs and monoidal transformations, Mat. Sb. (N.S.) 77 (119) (1968), 475-507. MR 41 \#3482

[MVW] C. Mazza, V. Voevodsky, and C. Weibel, Lecture notes on motivic cohomology, Preprint available at



[Org04] F. Orgogozo, Isomotifs de dimension inférieure ou égale á un, Manuscripta Mathematica Online First (2004).

[V. 00] V. Voevodsky, A. Suslin and E. M. Friedlander, Cycles, transfers, and motivic homology theories, Annals of Mathematics Studies, vol. 143, Princeton University Press, 2000.

[Voe00] V. Voevodsky, Triangulated Categories of Motives Over a Field, in VSF V. 00, pp. 188-254. 